Supporting Information

\title{
Surface Morphologies of Planar Ring Polyelectrolyte Brushes Induced by Trivalent Salts
}

\author{
Qing-Hai Hao, ${ }^{* \dagger}$ Jie Cheng, ${ }^{\dagger}$ Li-Xiang Liu, ${ }^{\dagger}$ Hong-Ge Tan,${ }^{\dagger}$ Tong Wei, ${ }^{\dagger}$ Li-Yan Liu, ${ }^{\dagger}$ \\ and Bing Miao* \\ ${ }^{\dagger}$ College of Science, Civil Aviation University of China, Tianjin 300300, China

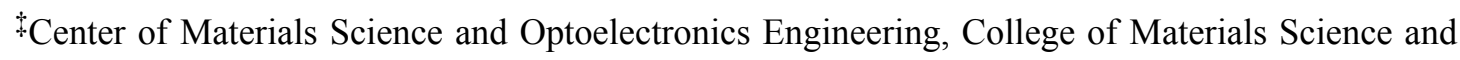 \\ Opto-Electronic Technology, University of Chinese Academy of Sciences, Beijing 100049, China
}




\section{S1. Model of the 'Equivalent' Linear Polyelectrolyte Brush}

In order to reveal the similarities and differences between the ring polyelectrolyte (PE) brush and its linear counterpart, we construct the linear PE brush model by cutting off the mid-bonds of the ring chains. The yielded linear PE brush is shown in Figure S1.

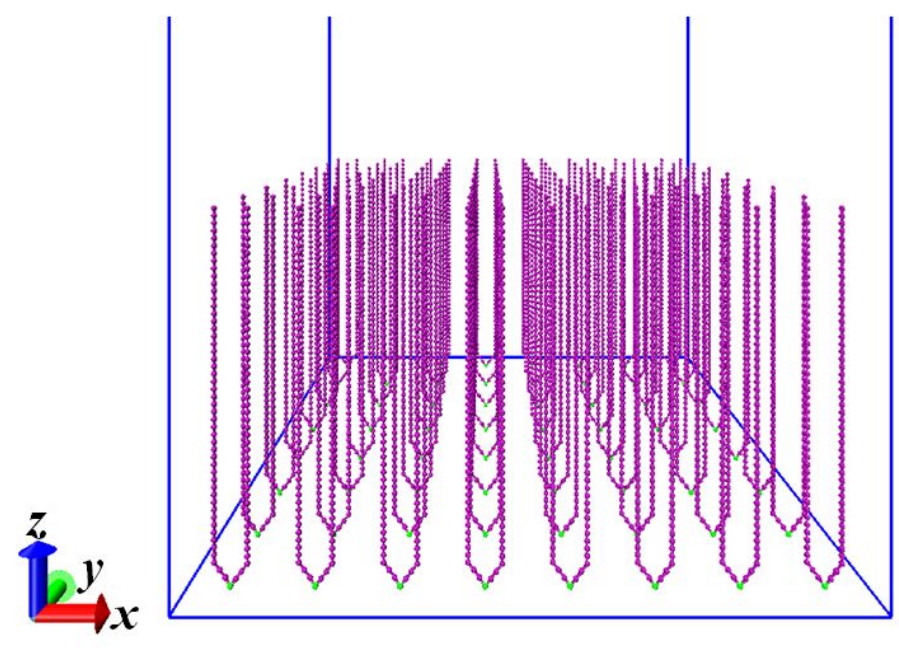

Figure S1. The snapshots of the simulation setup for linear PE brushes. Only the grafted points (green beads) and the monomers (purple beads) are shown for clarity.

\section{S2. The Effect of Trivalent Salt Concentration}

Using the linear model of planar brushes, the effect of trivalent salt concentration on the surface structures of linear PE brushes at grafting density $\sigma_{\mathrm{g}}=0.008$ under good and poor solvent conditions is investigated systematically. It is found that the vertical distributions of monomers of the linear PE brushes are similar to those of the ring PE brushes, as illustrated by the height of brush (demonstrated in Figure S2a) and the $z$-component of gyration radius (demonstrated in Figure S2b). 

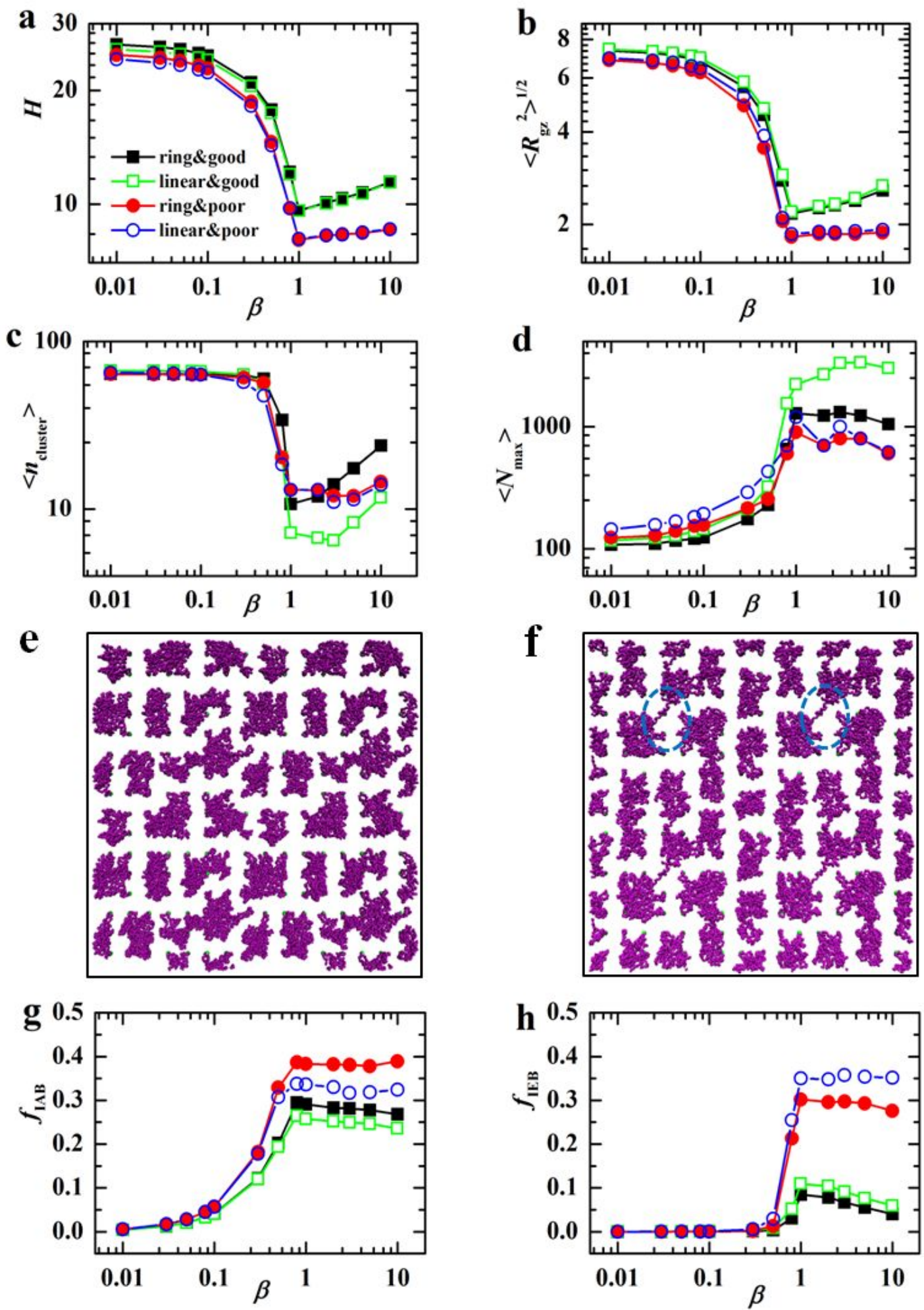

Figure S2. Comparisons of ring PE brush and its linear counterpart as a function of trivalent salt concentrations at grafting density $\sigma_{\mathrm{g}}=0.008$. (a) height of brush, (b) $z$-component of gyration radius, (c) number of pinned patches, (d) number of monomers in the largest patch, (e) top view of 
snapshot from simulations of ring PE brush under good solvent condition at $\beta=1.0, N_{\mathrm{R}}=100$, (f) top view of snapshot from simulations of linear PE brush under good solvent condition at $\beta=1.0$, degree of polymerization $N_{\mathrm{L}}=50,(\mathrm{~g})$ intra-chain bridging fraction of trivalent ions, and (h) inter-chain bridging fraction of trivalent ions. The inset ellipses in (f) are used to mark the two neighboring patches holding together through stretched linear PE chains. (e) and (f) are duplicated periodically along $x$ and $y$ directions for comparison.

The cluster analysis technique, ${ }^{1}$ which is able to identify monomers belonging to common clusters (pinned micelles) and measures various cluster properties, is employed for monitoring the lateral surface structure of ring and linear PE chains. The inter-monomer distance is used to determine whether a monomer belongs to a given cluster (pinned micelle). That is, if the interval distance of monomer $i$ to any monomer within the given cluster is less than or equal to $r_{\text {cutoff }}$, the monomer $i$ will be included in this cluster. Here, the $r_{\text {cutoff }}$ is selected as $r_{\text {cutoff }}=1.4$, corresponding to the first minimum in the monomer-monomer pair correlation function (see Figure S7). Based on the cluster analysis technique, the properties of pinned aggregates of ring and linear PE brushes are calculated, which are shown in Figure S2c and S2d.

As observed from Figure S2c and S2d, for the PE ring and linear brushes, the properties of lateral surface patterns are similar in poor solvent conditions at the whole salt conditions, while the main differences come from the high salt concentration regime of $\beta \geq 1.0$ in good solvent conditions. Specifically, it is found that, for high salts and good solvents, less lateral pinned patches are formed for linear PE brush compared to the case of ring PE brush, as shown in Figure S2c. This in turn 
leads to a larger patch size for the linear PE brush compared to the case of ring PE brush, which is demonstrated in Figure S2d.

This observed difference for the lateral surface structures between linear and ring PE brush in the simulation requires a careful theoretical explanation in the further studies. While the analytical model for the phase behavior of a ring PE brush is still lacking up to the present study, it might be developed from that for the linear PE brush as constructed in the main part Reference 45. In the present study, however, we provide a conjecture on the underlying reason for the difference based on our simulations. From the top views of pinned patches for the two cases shown in Figure S2e and S2f, we observe, as marked by the ellipses in Figure S2f, that a larger patch is realized by holding together neighboring patches through stretched linear PE chains, while this phenomenon is absent in the ring PE case. Therefore, the difference might be attributed to the different degrees of freedom for chain configuration in the cases of linear and ring brushes. Specifically, compared to the ring correspondence, linear chains can explore more spaces due to the relaxation of topology constraint for a ring chain. In the presence of high concentration of trivalent ions in good solvents, as known, strong electrostatic correlations result in the formation of lateral patches. Hence, the trivalent ions bridge the stretched linear PE chains from neighboring patches leading to the formation of patches of larger sizes for the linear PE brush. Due to the limited degree of freedom for ring chains, the above mechanism of larger patch formation is absent for the ring PE brush. This then explains the differences for the two types of brushes. We again note that this explanation is only based on the 
observed morphologies in our simulations and a detailed analysis shall resort to a specific analytical model for the ring PE brushes.

Our conjecture is further supported by the states of trivalent ions in the two different brush system, which is shown in Figure S2g and S2h, where it is observed that, compared to the ring PE brush case, more trivalent ions locate at the inter-chain bridging state for the linear PE case, which then induces the formation of larger patches as explained above.

\section{S3. The Effect of Charge Fraction}

The effect of charge fraction on the surface morphologies of ring and linear PE brushes is investigated for fixed grafting density $\sigma_{\mathrm{g}}=0.008$ and trivalent salt concentration $\beta=1.0$. Specifically, the charge fraction $f$ is selected as $f=0.1,0.2$, $0.33,0.5,0.67,0.8,0.9$, and 1.0 . The charges are distributed uniformly along the backbone of tethered PE chains. For example, every three monomers carry a negative charge for $f=0.33$ and every three monomers carry two negative charges for $f=0.67$, respectively.

To characterize the difference of chain shape between ring and linear PE brushes, the extent of asphericity $\delta$ of grafted chain is calculated, which is defined as, ${ }^{2-4}$

$$
\delta=\sum_{i=1}^{3} \frac{\left(\lambda_{i}-\bar{\lambda}\right)^{2}}{6 \bar{\lambda}^{2}}, \bar{\lambda}=\sum_{i=1}^{3} \frac{\lambda_{i}}{3}
$$

where $\lambda_{i}$ are the eigenvalues of the inertia tensor. $\delta=0$ is for a perfect sphere and deviation from $\delta=0$ indicates the extent of anisotropy. The dependence of asphericity on the charge fraction is plotted in Figure S3b. 

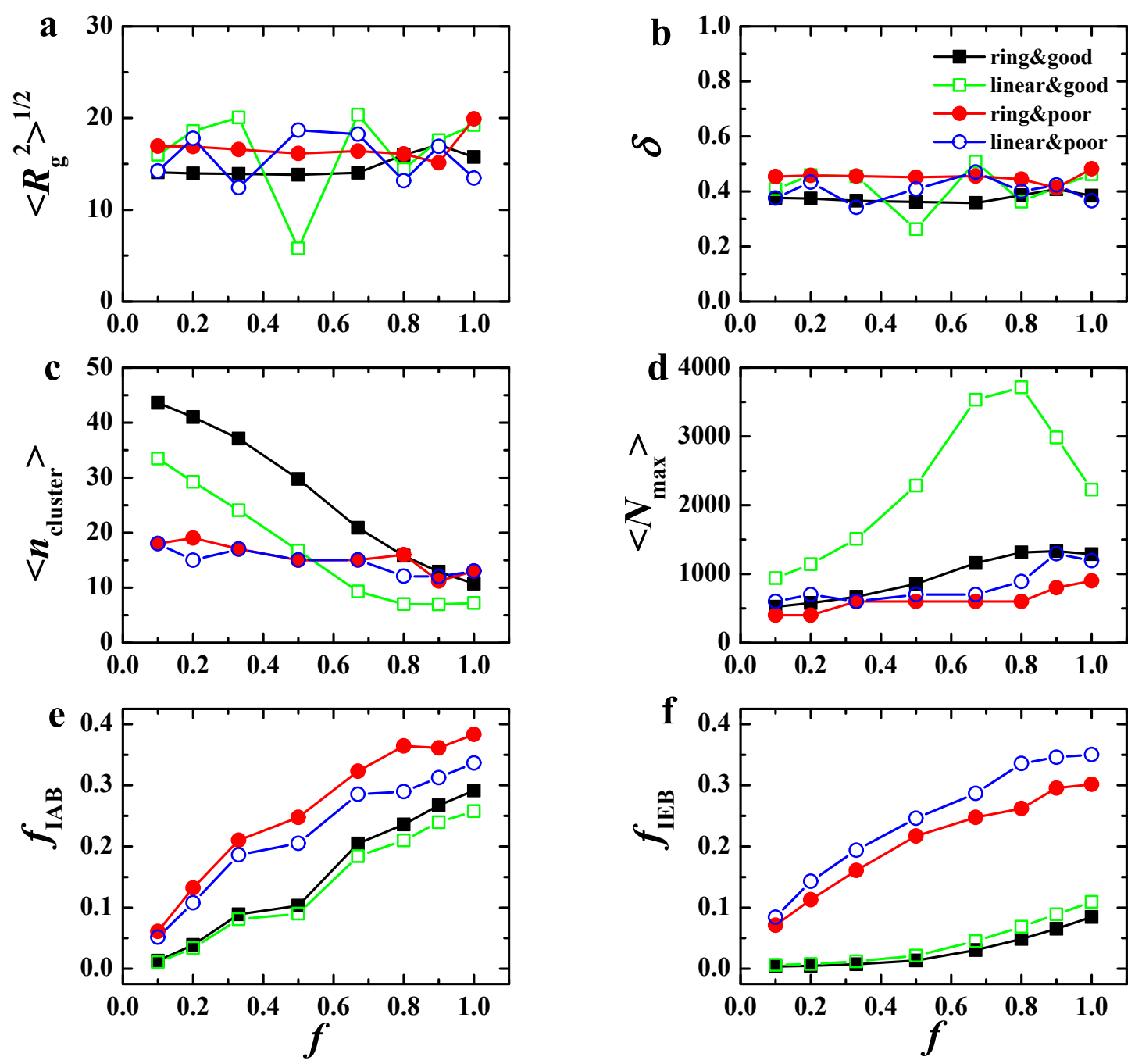

Figure S3. Comparisons of ring PE brush and its linear counterpart as a function of charge fraction at grafting density $\sigma_{\mathrm{g}}=0.008$. (a) gyration radius, (b) asphericity, (c) number of pinned patches, (d) number of monomers in the largest patch, (e) intra-chain bridging fraction of trivalent ions, and (f) inter-chain bridging fraction of trivalent ions. The solid and open symbols represent the cases of ring and linear PE brushes respectively.

As shown in Figure $\mathrm{S} 3 \mathrm{a}$ and $\mathrm{S} 3 \mathrm{~b}$, while the gyration radius $R_{\mathrm{g}}$ and the asphericity $\delta$ are almost independent of the charge fraction for the ring PE brush, oscillatory profiles are observed for the linear PE case. The vertical distributions of monomers for the ring and linear PE brushes are similar to each other (not shown here), while 
again the lateral structures behave differences, as shown through the effect of charge fraction on the number of pinned patches and the number of monomers in the largest patch in Figure S3c and S3d respectively. Consistent with the observation in Figure S2e and S2f, it is found that the obvious differences between the two brush system occur at good solvent conditions and the linear PE brush admits less patches and larger patch size, which might be, as explained above, due to the more degrees of freedom for configuration fluctuations available for the linear PE chains rendering the trivalent ion inter-chain bridging induced formation of larger patches. Figure S3e and S3f illustrates the states of trivalent ions, which further confirms that, compared to the ring PE brush case, there exit more inter-chain bridging trivalent ions in the linear PE brush case, which then induces the formation of larger patches for the linear brush system.

\section{S4. The Effect of Degree of Polymerization}

The effect of degree of polymerization is investigated by changing the chain lengths of the ring and linear PE brushes for fixed trivalent salt concentration $\beta=1.0$ and grafting density $\sigma_{\mathrm{g}}=0.008$. The chain lengths are chosen as $N_{\mathrm{R}}=20,40,60, \cdots$, 200 , and the 'equivalent' linear chain lengths are $N_{\mathrm{L}}=10,20,30, \cdots, 100$. The vertical monomer distributions, illustrated by the height of brush and $z$-component radius of gyration, are similar for the ring and linear PE brushes, as shown in Figure S4a and $\mathrm{S} 4 \mathrm{~b}$. 

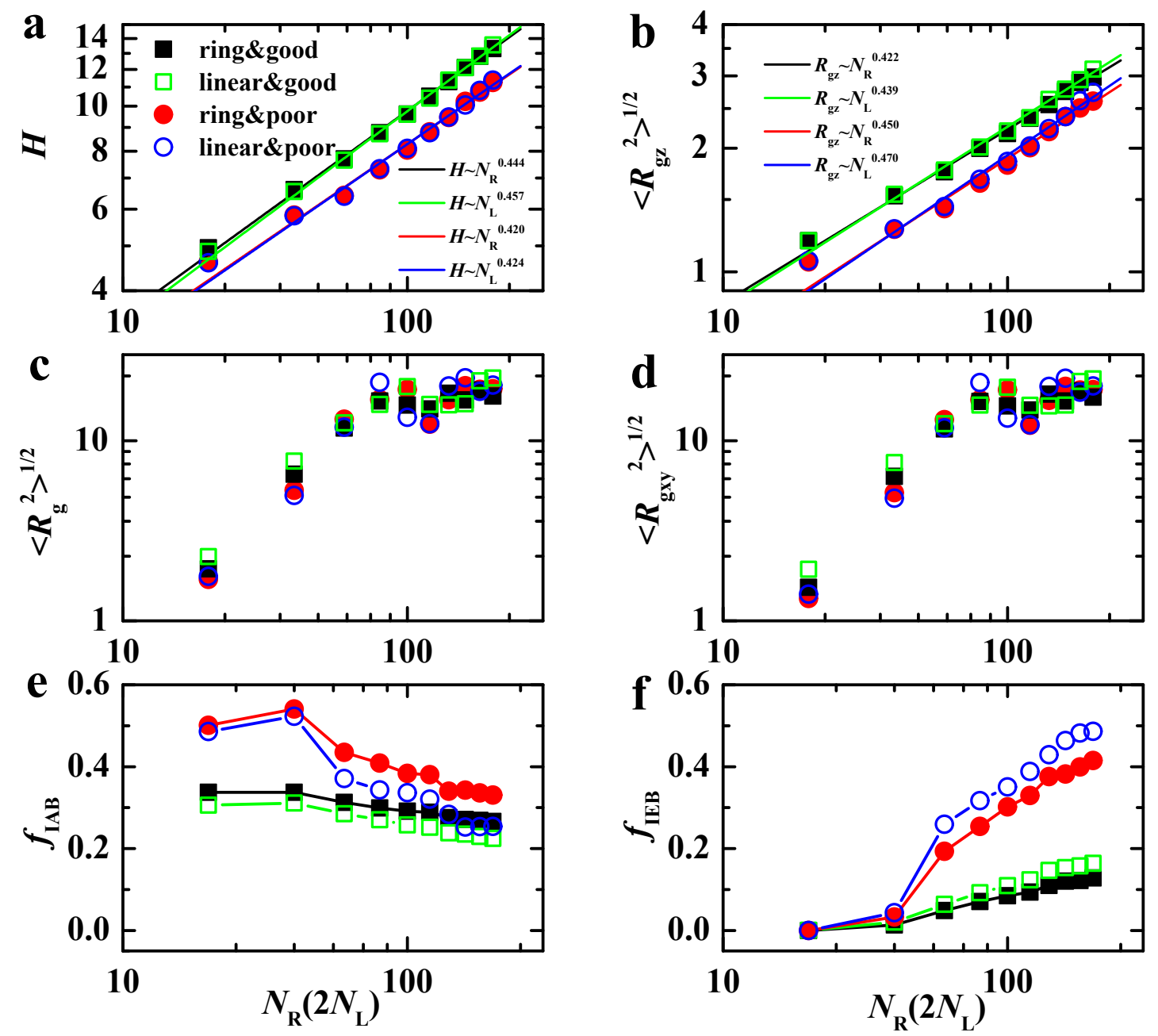

Figure S4. Heights of ring (solid symbols) and linear (open symbols) PE brushes (a), $z$-component radius of gyration (b), gyration radius (c), $x-y$ plane component radius of gyration (d), fractions of trivalent ions in the intra-chain bridging state (e), and fractions of trivalent ions in the inter-chain bridging state (f) as a function of degree of polymerization at grafting density $\sigma_{\mathrm{g}}=0.008$ under good and poor solvent conditions.

As shown in Figure $\mathrm{S} 4 \mathrm{a}$ and $\mathrm{S} 4 \mathrm{~b}$, both the height of the brush $H$ and the $z$-component $R_{\mathrm{gz}}$ of gyration radius show a scaling relation as $H \sim N_{\mathrm{R}}{ }^{v}\left(\sim N_{\mathrm{L}}{ }^{v}\right)$ and $R_{\mathrm{gz}}$ $\sim N_{\mathrm{R}}{ }^{v}\left(\sim N_{\mathrm{L}}{ }^{v}\right)$. The exponential fitting lines are almost overlapped for the ring and linear PE brushes under good and poor solvent conditions, indicating the similarity of vertical distributions of monomers for the two cases. 
The gyration radius $R_{\mathrm{g}}$ and the in-plane component $R_{\mathrm{gxy}}$ can characterize the lateral distribution of ring and linear PE brushes, which are shown in Figure S4c and S4d. As found, $R_{\mathrm{g}}$ and $R_{\mathrm{gxy}}$ increase with the degree of polymerization in $N_{\mathrm{R}}\left(2 N_{\mathrm{L}}\right) \leq$ 80 , and fluctuate in the large polymerization degree regime of $N_{\mathrm{R}}\left(2 N_{\mathrm{L}}\right) \geq 80$, which corresponds to the transition of surface morphologies from individual mushrooms to pinned patch structures. The transition point $N_{\mathrm{R}}{ }^{*}$ is around $80\left(N_{\mathrm{L}}{ }^{*} \approx 40\right)$, which is similar to the scaling prediction of linear PE brushes. ${ }^{4}$ Furthermore, the difference of lateral distributions of monomers between the ring and linear PE brushes can also be observed in Figure S4c and S4d. Compared to the ring PE brush, there exist more inter-chain bridging trivalent ions, as shown in Figure S4e and S4f, which leads to the formation of larger patches in the linear PE brush system, as explained above.

\section{S5. Supplementary Results for the Ring PE Brushes}

\section{S5.1 Vertical Distributions of Ions}

The vertical distributions of trivalent salt cations are plotted in Figure S5a (good solvent) and S5b (poor solvent). For $\beta<1.0$, independent of solvent condition, trivalent salt cations accumulate in the interior of the ring PE brush owing to the stronger electrostatic attraction between them and charged monomers, which corresponds to a relatively large value of $\rho_{\mathrm{sc}}(z)$ near the grafting surface (black and red curves). The ion-exchange phenomenon occurs first in the inner layer of the brush, which is due to the fact that releasing counterions in the inner region to outside of the brush results in larger translational entropy of counterions. At $\beta=1.0$, as expected, the distribution of trivalent cations (blue curves in Figure S5a and S5b) demonstrates 
almost the same profiles that of monomers plotted in Figure 5a and 5b, however, the magnitude is just one third of that of the monomer distribution profile, which is due to the requirement of global electrostatic neutrality. For $\beta>1.0$, the value of $\rho_{\mathrm{sc}}(z)$ of $\beta=$ 10.0 (green curves in Figure S5a) is slightly larger than that of $\beta=1.0$ (blue curve in Figure S5a) in the ring brush region, which indicates the re-entrance phenomena under good solvent condition. However, the curves of $\rho_{\mathrm{sc}}(z)$ in $\beta \geq 1.0$ are approximately overlapping within the brush layer (green and blue lines in Figure S5b), indicating the absence of re-entrance process in the poor solvent. The excess salt cations disperse uniformly outside the brush, which leads to a plateau profile in good and poor solvents (green curves in Figure S5a and S5b).
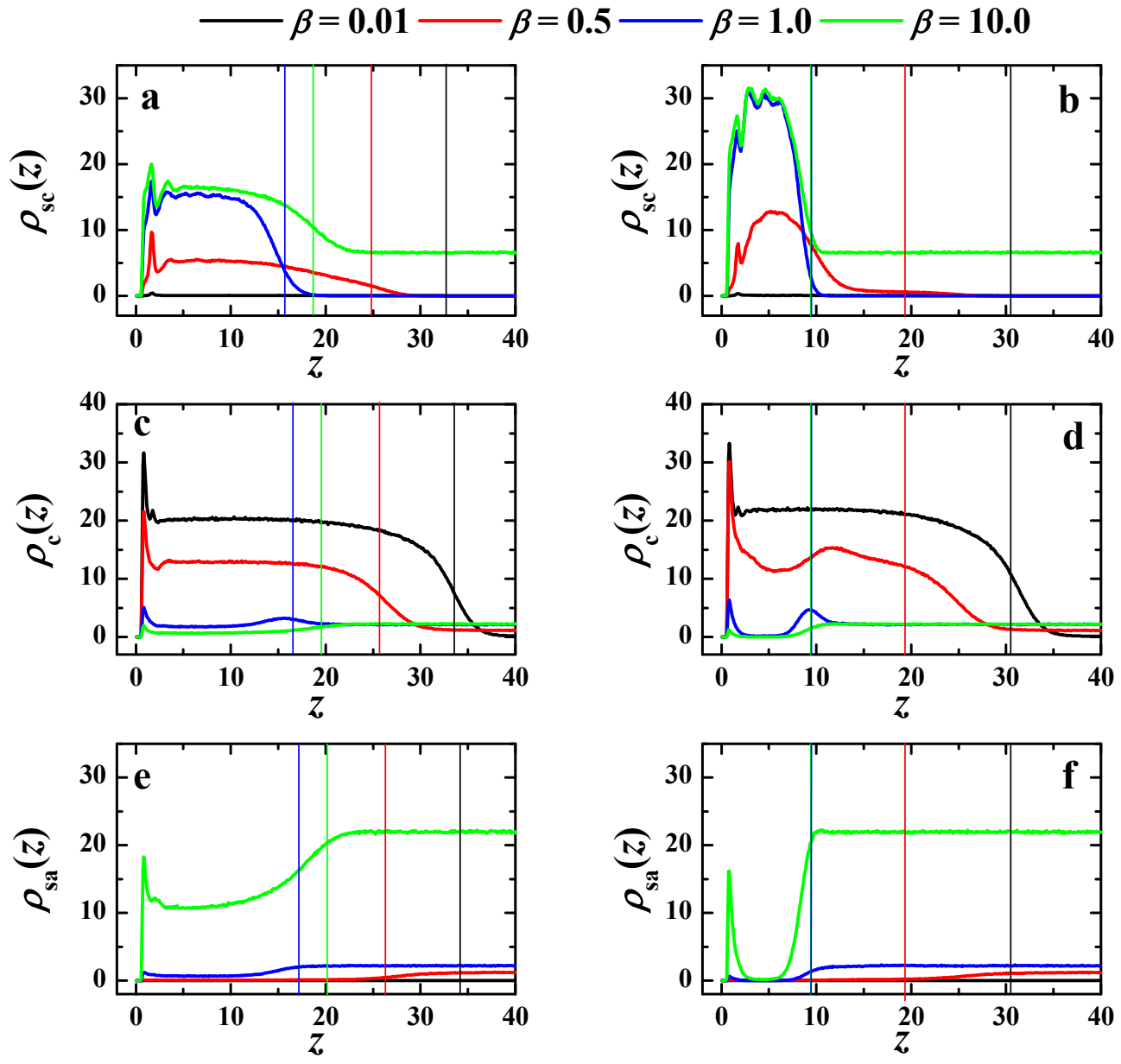
Figure S5. The distributions of trivalent salt cations (a) and (b), monovalent counterions (c) and (d), monovalent salt anions as a function of the distance from the substrate in good solvent (left panels) and poor solvent (right panels) at high grafting density $\sigma_{\mathrm{g}}=0.04$. For clarity, profiles of only four salt concentrations are plotted. The vertical lines in each panel are employed to display the height of the ring PE brush. The style and color of the vertical lines are the same as those of the distribution profiles.

The vertical distributions of monovalent counterions are illustrated in Figure S5c and S5d. At $\beta=0.01$, almost all counterions are distributed within the brush layer and neutralize the polyion charge. The osmotic pressure of monovalent counterions leads the ring brush to adopt a swollen morphology regardless of solvent quality. With the increase of trivalent salt concentration, more counterions are substituted by trivalent salt cations and disperse uniformly outside the brush. For $\beta>1.0$, only a few counterions are confined close to the substrate. Figure S5e and S5f plot the vertical distribution of monovalent salt anions, where it is found that salt anions are driven outside the brush layer because of the electrostatic repulsion, irrespective of the solvent condition for $\beta \leq 1.0$. For $\beta>1.0$, owing to the re-entrance phenomenon of trivalent salt cations in good solvents, a certain amount of salt anions is attracted into the ring brush. Whereas in poor solvents, the re-entrance is absent and only few salt anions are confined near the grafting surface.

\section{S5.2 Distributions of Bond Orientation}

In order to address the distributions of bond orientation in the brush, the second Legendre polynomial $P_{2}\left(\theta_{i}\right)$ is calculated, ${ }^{5,6}$ 


$$
P_{2}\left(\theta_{i}\right)=\frac{1}{2}\left(3\left\langle\cos ^{2} \theta_{i}\right\rangle-1\right)
$$

Clearly, $P_{2}\left(\theta_{i}\right)=0$ corresponds to a random distribution of bond orientation, $P_{2}\left(\theta_{i}\right)>0$ indicates a preference of the bond orientation perpendicular to the grafting surface, and $P_{2}\left(\theta_{i}\right)<0$ indicates a bond orientation parallel to the grafting surface. The evolution of $P_{2}\left(\theta_{i}\right)$ of bonds along the ring chain are demonstrated in Figure S6.
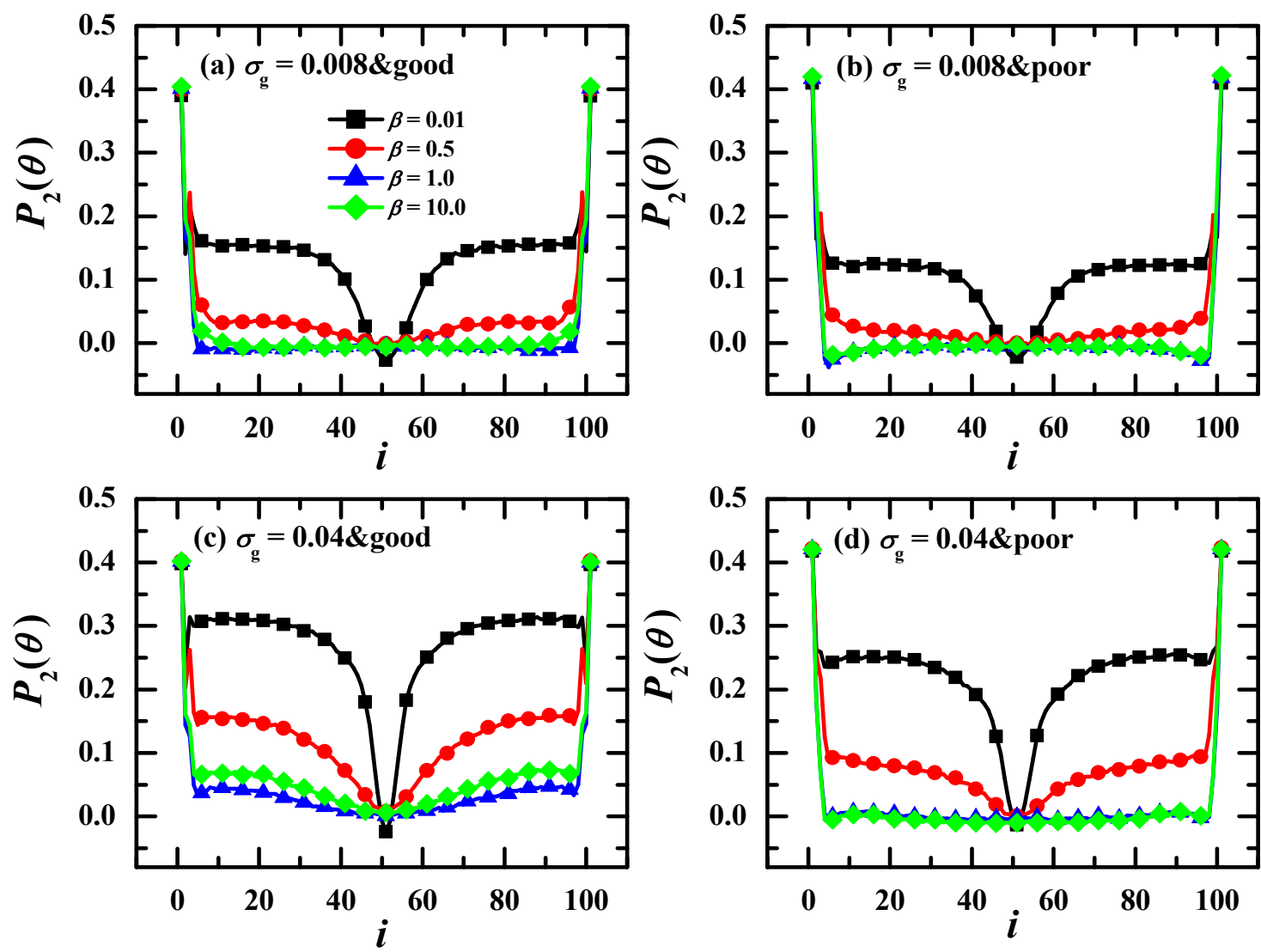

Figure S6. Average bond orientations $P_{2}\left(\theta_{i}\right)$ versus the bond sequential number $i$ in different trivalent salt solutions. (a) $\sigma_{\mathrm{g}}=0.008$ and in good solvent, (b) $\sigma_{\mathrm{g}}=0.008$ and in poor solvent, (c) $\sigma_{\mathrm{g}}=0.04$ and in good solvent, (d) $\sigma_{\mathrm{g}}=0.04$ and in poor solvent.

As shown in Figure S6a, the average bond orientation $P_{2}\left(\theta_{i}\right)$ close to the grafting surface ( $i \leq 5$ and $i \geq 95$ ) has a relative large positive value and decreases sharply regardless of salt concentration, indicating the tendency of perpendicular orientation 
of these bonds, which reflects the dominant role of excluded volume interaction of substrates in the interior of ring PE brushes. A pronounced valley located around the mid-monomer of the ring chains is observed at $\beta=0.01$ (solid squares in Figure S6a), and the $P_{2}\left(\theta_{i}\right)$ in the valley has a negative value close to zero, which means that the bonds close to the mid-monomer prefer to be parallel to the substrate. For $\beta=0.5$, the valley near the mid-monomer becomes shallow (see filled circles in Figure S6a), which results from the shrink of the ring PE chains induced by the trivalent ions. With further increasing salts to $\beta \geq 1.0$, the valley disappears and a plateau profile of $P_{2}\left(\theta_{i}\right)$ (close to zero) is observed for the mid part of the monomers, indicating that the orientations of these middle bonds are randomly distributed inside the brush. For the low grafting density $\sigma_{\mathrm{g}}=0.008$ under poor solvents conditions plotted in Figure S6b, the valleys of $\beta=0.01$ and 0.5 for the middle part of ring chains are slightly shallower than those in good solvents, which corresponds to a relatively tight conformation of PE chains induced by the hydrophobicity in poor solvents. For $\beta \geq 1.0$, the difference between good and poor solvent conditions at low grafting density is that the $P_{2}\left(\theta_{i}\right)$ of $i$ $=5,95$ are negative, indicating parallel configurations of these bonds.

For the high grafting density $\sigma_{\mathrm{g}}=0.04$ under good solvent condition, as depicted in Figure S6c, the valley of $\beta=0.01$ becomes pronounced and its width becomes narrower compared with that of low grafting density (filled squares in Figure S6a). With increasing salt concentration, the valley near the mid-monomer is always observed in good solvents, which originates from the non-uniform stretching of the middle part of ring chains. Moreover, the $P_{2}\left(\theta_{i}\right)$ of $\beta=10.0$ adopts a slightly larger 
value than that of $\beta=1.0$, which confirms the re-entrance phenomenon where re-swelling of the ring brushes is induced by overcompensated multivalent ions under good solvent condition. As shown in Figure S6d, the valley of $\beta<1.0$ in poor solvents becomes less pronounced compared to that in good solvents (Figure S6c). Furthermore, the valley disappears for $\beta \geq 1.0$ and profiles of $P_{2}\left(\theta_{i}\right)$ are overlapping, which confirms the absence of re-entrance phenomenon in poor solvents.

\section{S5.3 Pair Correlation}
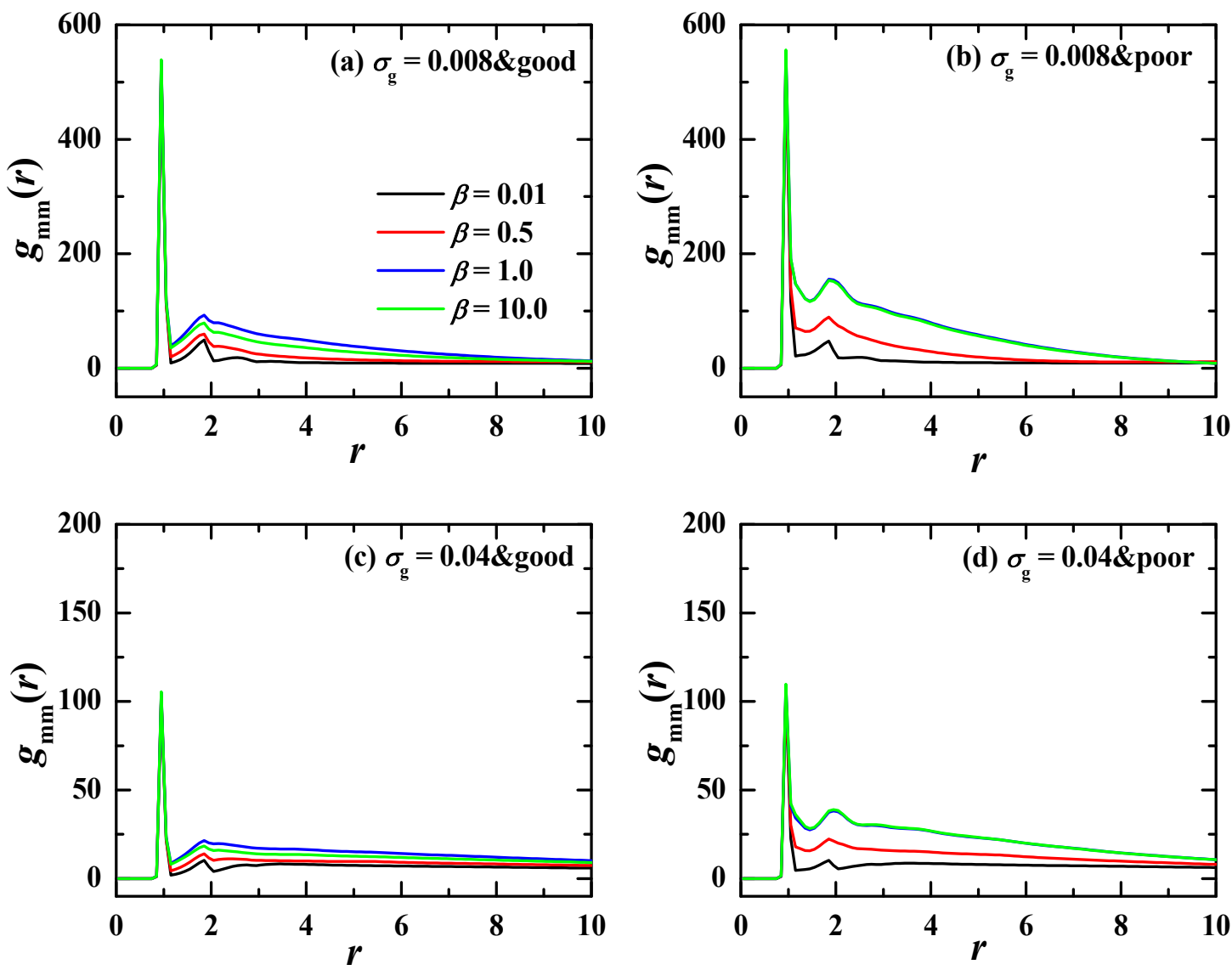

Figure S7. The pair correlation functions of monomer-monomer in different trivalent salt solutions. (a) $\sigma_{\mathrm{g}}=0.008$ and in good solvent, (b) $\sigma_{\mathrm{g}}=0.008$ and in poor solvent, (c) $\sigma_{\mathrm{g}}=0.04$ and in good solvent, (d) $\sigma_{\mathrm{g}}=0.04$ and in poor solvent.

To further clarify the local structuring of the ring PE brush, we calculate the pair 
correlation functions (PCF). The monomer-monomer PCF in different trivalent salt concentrations under good and poor solvent conditions are illustrated in Figure S7. In Figure S7a, a narrow primary peak and a distinct secondary peak can be observed at $\beta$ $=0.01$ (black curve in Figure S7a). The height of the secondary peak as well as the global amplitude of the monomer-monomer correlation profile gets larger with the increase of trivalent salt concentration for $\beta \leq 1.0$, which indicates that the lateral heterogeneity occurs in a larger length scale with the addition of trivalent salt ions. We note that the multivalent-ion-induced order under good solvent conditions was also observed for the linear PE brushes. ${ }^{4,7}$ When the charge of added trivalent salts exceeds that of monomers, the long-range order weakens under good solvent conditions due to the re-entrance of trivalent salt ions (green curve in Figure S7a). In the case of low grafting density $\sigma_{\mathrm{g}}=0.008$ in poor solvents, as shown in Figure S7b, both the nearest neighbor correlation and the long-range correlation become pronounced compared to those in good solvents for fixed $\beta$ due to the coupling of electrostatic correlation of trivalent ions with the hydrophobic effect of solvent. Moreover, once the trivalent salt concentration reaches $\beta=1.0$, the PCF profiles of monomer-monomer are overlapping for further increasing trivalent salt concentrations under poor solvent conditions, which again indicates that the re-entrance phenomenon is absent in poor solvents. For the high grafting density $\sigma_{\mathrm{g}}=0.04$ shown in lower panels of Figure S7, it is found that the profiles of the whole monomer-monomer PCF become weaker compared to those at low grafting density regardless of solvent quality, which is due to the enhanced excluded volume effect with the increase of 
grafting density.

\section{References}

(1) Rapaport, D. C. The Art of Molecular Dynamics Simulation, 2nd ed; Cambridge University Press: 2004.

(2) Aronovitz, J. A.; Nelson, D. R. Universal features of polymer shapes. J. Phys. (Paris) 1986, 47, 1445-1456.

(3) Hyeon, C.; Dima, R. I.; Thirumalai, D. Size, shape, and flexibility of RNA structures. J. Chem. Phys. 2006, 125, 194905.

(4) Liu, L.; Pincus, P. A.; Hyeon, C. Heterogeneous Morphology and Dynamics of Polyelectrolyte Brush Condensates in Trivalent Counterion Solution. Macromolecules 2017, 50, 1579-1588.

(5) He, S. Z.; Merlitz, H.; Su, C. F.; Wu, C. X. Static and Dynamic Properties of Grafted Ring Polymer: Molecular Dynamics Simulation. Chin. Phys. B 2013, 22, 016101.

(6) Wan, W. B.; Lv, H. H.; Merlitz, H.; Wu, C. X. Static and Dynamic Properties of Polymer Brush with Topological Ring Structures: Molecular Dynamic Simulation. Chin. Phys. B 2016, 25, 106101.

(7) Jackson, N. E.; Brettmann, B. K.; Vishwanath, V.; Tirrell, M.; de Pablo, J. J. Comparing Solvophobic and Multivalent Induced Collapse in Polyelectrolyte Brushes. ACS Macro Lett. 2017, 6, 155-160. 